\title{
Neumoperitoneo en un paciente en tratamiento con diálisis peritoneal automática: informe de un caso
}

\author{
Natalia Formento-Marín, María José Cintora-Querol, María Soriano-Angulo \\ Servicio de Nefrología. Hospital Universitario Miguel Servet. Zaragoza. España
}

Como citar este artículo:

Formento-Marín N, Cintora-Querol MJ, Soriano-Angulo M. Neumoperitoneo en un paciente en tratamiento con diálisis peritoneal automática: informe de un caso. Enferm Nefrol. 2020 0ct-Dic;23(4):406-410

\section{Resumen}

Descripción del caso: caso de neumoperitoneo en un paciente con tratamiento en diálisis peritoneal automática con día seco. Pese a que el neumoperitoneo no es una complicación grave, puede alterar la calidad de vida del paciente, por lo que es necesario investigar su origen. Tras revisar la bibliografía existente, encontramos un único caso de neumoperitoneo en terapia de diálisis peritoneal automática y se han reportado un mayor número de casos de esta complicación en pacientes con diálisis peritoneal continua ambulatoria, todos ellos debido a errores en la técnica. Tras la valoración de enfermería, el paciente identificó algunos errores en la técnica como los posibles causantes de esta complicación.

Descripción del plan de cuidados: El plan de cuidados consistió en descartar otras complicaciones de la diálisis peritoneal como obstrucción de catéter o peritonitis, evaluar los posibles errores del paciente en la técnica procediendo a su reentrenamiento y ofrecer apoyo emocional al paciente y la familia. La evaluación del plan de cuidados a la semana y al mes fue favorable.

Conclusiones: Como conclusión, hay que destacar la importancia de realizar un entrenamiento personali-

\section{Correspondencia:}

Natalia Formento Marín

E-mail: natalia.formento7@gmail.com zado en cada paciente, dependiendo del tipo de terapia prescrita y las potenciales complicaciones para que el paciente las conozca y así poder prevenir su aparición.

PALABRAS CLAVE: diálisis peritoneal; neumoperitoneo; enfermería en nefrología; nefrología; informes de casos.

\section{Pneumoperitoneum in a patient receiving automatic peritoneal dialysis: a case report}

\section{Abstract}

Case description: Case of pneumoperitoneum in a patient on automatic peritoneal dialysis with a dry day. While pneumoperitoneum is not a serious complication may alter the quality of life of the patient, so it is necessary to determine its origin. After reviewing the existing bibliography, we found a case report of pneumoperitoneum on automatic peritoneal dialysis therapy. In addition, other cases with this complication in patients with continuous ambulatory peritoneal dialysis have been reported, all of them because of errors in the technique. After the nursing assessment, the patient identified some errors in the technique as the possible causes of this complication.

Description of the care plan: The care plan focused on ruling out other complications of peritoneal dialysis such as catheter obstruction or peritonitis, evaluating possible errors in the technique made by the patient, 
proceeding with their retraining, and offering emotional support to the patient and the family. The evaluation of the care plan at one week and one month was favorable.

Conclusions: It should be emphasized the importance of personalized training depending on the type of therapy prescribed and the potential complications, so that the patient knows them and can prevent their appearance.

KEYWORDS: peritoneal dialysis; pneumoperitoneum; nephrology nursing, nephrology, case reports.

\section{Introducción}

El neumoperitoneo es una complicación que se manifiesta por la presencia de aire libre en la cavidad abdominal, siendo el $90 \%$ de los casos provocado por una perforación de una víscera hueca. En el caso de los pacientes en diálisis peritoneal (DP), la presencia de un catéter en el peritoneo que pone en contacto dicha cavidad con el ambiente puede conllevar el paso del aire siendo la causa de esta complicación. La bibliografía reporta que entre un $10-34 \%$ de los pacientes en diálisis peritoneal continua ambulatoria (DPCA) presentan aire subdiafragmático, sin relevancia clínica en la mayoría de los casos, resolviéndose sin complicaciones y siendo su tratamiento conservador ${ }^{1-3}$.

Ante la presencia de un neumoperitoneo siempre se ha de buscar el origen causal que la provoca. Existen casos reportados de neumoperitoneo producidos en la inserción del catéter peritoneal ${ }^{2}$ o por determinados microorganismos infecciosos que originan peritonitis ${ }^{3,4}$. También sen han reportado casos debido a fallos en la realización de la técnica de DP. En la mayoría de los casos de neumoperitoneo debido a errores en la técnica ocurren en terapia DPCA, por un inadecuado purgado de las líneas del sistema ${ }^{3,5-7}$. Tan sólo hemos encontrado un caso, descrito en el año 1986, de neumoperitoneo con DPA ${ }^{8}$.

Pese a que el neumoperitoneo no es una complicación grave, puede alterar la calidad de vida del paciente, por lo que es necesario investigar su origen y si se debe a errores en la realización de la técnica es imprescindible el reentrenamiento del paciente para prevenir nuevos episodios.

\section{Presentación del caso}

Presentamos el caso de un paciente en tratamiento en diálisis peritoneal automática (DPA), desde hace dos años en nuestro servicio, que acude a urgencias al presentar un episodio de neumoperitoneo.

Varón de 33 años, con antecedentes de hipertensión, dislipemia, insuficiencia renal crónica desde el nacimiento secundaria a una uropatia obstructiva, trasplantado renal a la edad de 7 años con nefropatía crónica del injerto. Durante la inserción del catéter peritoneal cursó con un edema agudo de pulmón y un SCASEST precisando la colocación de de 5 stent tras la colocación. Intervenido quirúrgicamente de hernioplastia inguinal izquierda como consecuencia del tratamiento en diálisis peritoneal. Episodios de hemorragias digestivas altas por lesiones vasculares en duodeno y hemoptisis con embolización bronquial.

Tratamiento mediante DPA nocturna desde hace 2 años con Cicladora de Baxter ${ }^{\circledR}$ HomeChoicePro con una programación de terapia tidal del $90 \%$ con drenajes completos cada 2 ciclos, con un tiempo de terapia de $7 \mathrm{~h}$, con un volumen total de $8.500 \mathrm{cc}$ con 4 ciclos y un volumen de infusión de 2.000 cc cada uno, con una última infusión de 200 cc (día seco).

Acude al servicio de Urgencias por sensación de dolor súbito en ambos hombros y reborde costal derecho durante la sesión de diálisis peritoneal esa misma noche. $\mathrm{A}$ la llegada al servicio presenta una TA de $157 / 100 \mathrm{mmHg}$, $\mathrm{FC}$ de $94 \mathrm{lpm}, \mathrm{T}^{\mathrm{a}}$ axilar de $37^{\mathrm{a}} \mathrm{C}$ y saturación de oxígeno del $98 \%$. La exploración cardiaca y pulmonar fue normal, abdomen blando y depresible sin signos de irritación peritoneal y ligeros edemas en extremidades inferiores.

Tras ser valorado en el servicio de urgencias se solicitó una analítica de sangre (sin hallazgos relevantes) y una radiografía de tórax (Figura $\mathbf{1}$ y Figura 2) donde se describen una imagen de densidad gas subdiafragmático sugestiva de neumoperitoneo. Tras el alta de los Servicios de Urgencias es derivado al Servicio de Diálisis Peritoneal para su valoración.

\section{Valoración enfermera}

Para la realización del plan de actuación enfermera se ha seguido el modelo de valoración de patrones funcionales de Marjory Gordon. Se realizó la valoración enfermera del paciente a la llegada a nuestra Unidad fue la siguiente: 


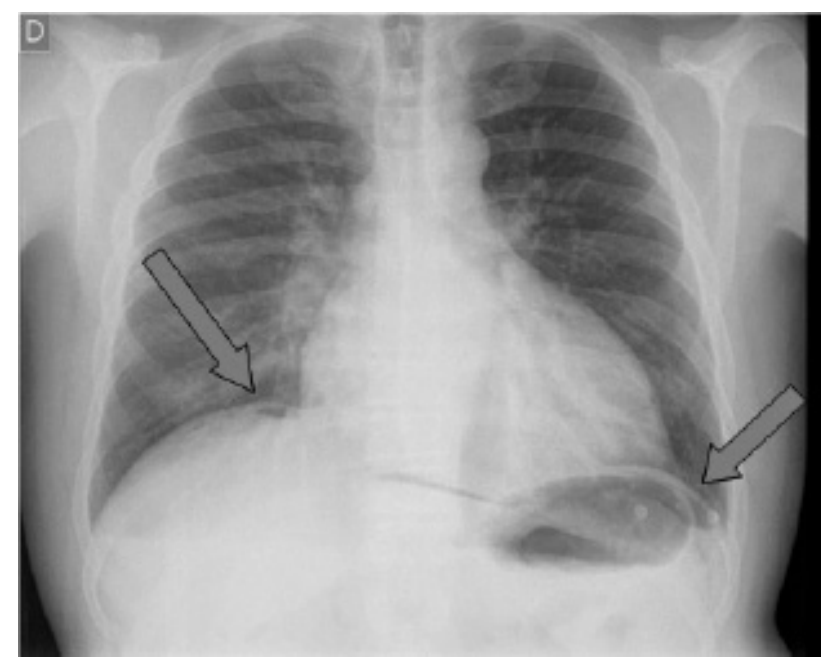

Figura 1. Radiografía anteroposterior de tórax.

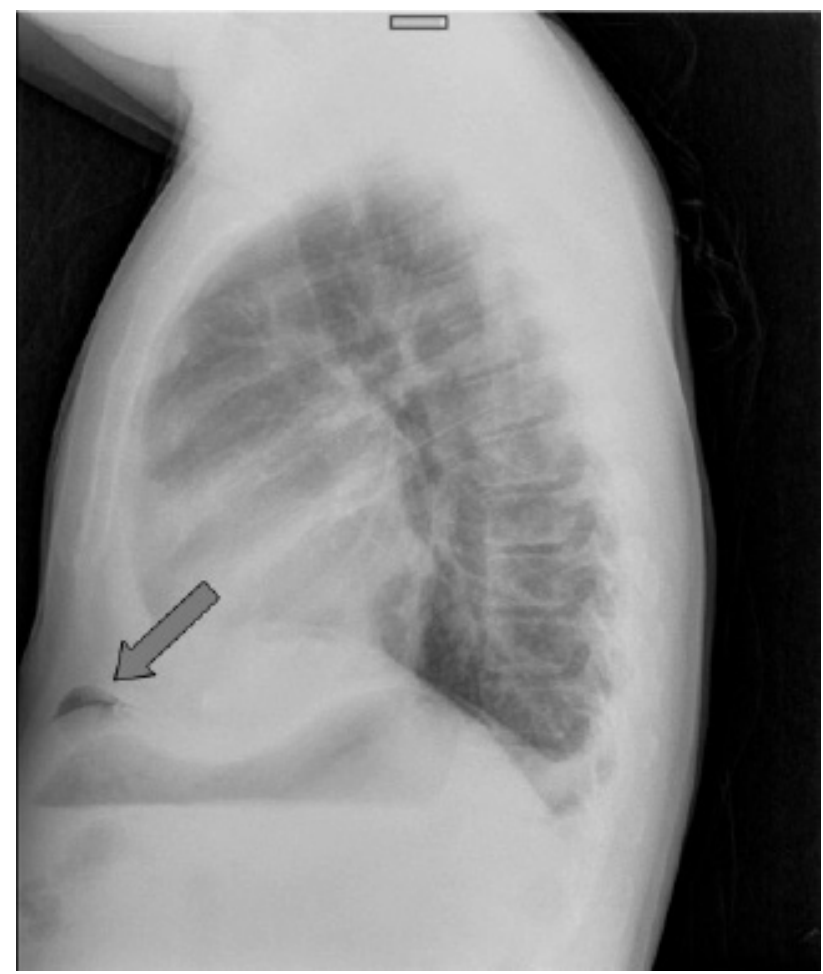

Figura 2. Radiografía lateral de tórax.

\section{- Patrón 1: Percepción-manejo de la salud}

Paciente sin alergias medicamentosas conocidas, con adecuada adherencia al tratamiento prescrito tanto en la medicación como en la terapia DPA, no hábitos tóxicos, con numerosos ingresos hospitalarios en diferentes ocasiones por cuadros de diarreas, hemorragias digestivas altas y hemoptisis sin incidencias en la DPA.

\section{- Patrón 2: Nutricional-metabólico}

No aumento de peso significativo respecto a últimos controles, actualmente con sobrepeso (IMC de 27,11), control adecuada de la ingesta de líquidos, no edemas. Realiza una dieta según las recomendaciones del personal sanitario.

\section{- Patrón 3: Eliminación}

Adecuado hábito intestinal sin estreñimiento. Sin incidencias en el funcionamiento del catéter peritoneal. Conservación adecuada de la función renal residual (1.900 ml/24h).

\section{- Patrón 4: Actividad-ejercicio}

Independiente para las actividades básicas de la vida diaria (ABVD) y actividades instrumentales de la vida diaria (AIVD). No realiza ejercicio habitualmente, habito sedentario. Sensación de disnea y plenitud con terapia DPA desde aumento del volumen de infusión en la última prescripción (2.000 cc por ciclo).

\section{- Patrón 5: Sueño-descanso}

Refiere sensación de plenitud con volúmenes de infusión actuales lo que en ocasiones interrumpe su descanso nocturno.

\section{- Patrón 6: Cognitivo-perceptual}

Orientado en espacio-tiempo. Miopía corregida con lentes, sin otras alteraciones sensoriales. Sin dificultades para el aprendizaje, reflexiona y detecta posibles fallos en la realización de la técnica que hayan conllevado el desarrollo de un neumoperitoneo. Presencia de dolor en zona costal que se irradia hacia los hombros.

\section{- Patrón 7: Autopercepción-autoconcepto}

Refiere ansiedad ocasional relacionada con las dificultades de afrontamiento de sus problemas de salud por parte de sus padres.

\section{- Patrón 8: Rol-relaciones}

Tiene grupo de amigos. Vive con sus padres, los cuales tienen una actitud excesivamente protectora con él según el paciente.

\section{- Patrón 9: Sexualidad-reproducción} No valorado.

\section{- Patrón 10: Adaptación-tolerancia al estrés}

Apoyo familiar, en ocasiones los padres presentan ansiedad ante los continuos ingresos de su hijo. El paciente maneja bien su situación emocional y utiliza el humor para ello.

\section{- Patrón 11: Valores-creencias}

No valorado. 


\section{Descripción del plan de cuidados?:}

El plan de cuidados se elaboró utilizando las taxonomías NANDA para los diagnósticos de enfermería, NOC para los resultados de enfermería y NIC para las intervenciones enfermeras (ver Tabla $\mathbf{1}$ ).

\section{Discusión}

Este informe de casos pone de manifiesto lo inusual que son los neumoperitoneos en la DPA. Existen muy pocos casos reportados en la literatura científica. Sin embargo, encontramos la descripción de un caso en DPA

Tabla 1. Plan de cuidados individualizado con taxonomías NANDA, NOC y NIC.

\begin{tabular}{|c|c|c|}
\hline NANDA & NOC & NIC \\
\hline $\begin{array}{l}\text { [00132] Dolor agudo. } \\
\text { Características definitorias: } \\
\text { - Autoinforme de intensidad del dolor } \\
\text { usando escalas estandarizadas de } \\
\text { valoración del dolor relacionado con } \\
\text { agentes lesivos físicos (indicando } \\
\text { una puntuación de } 7 \text { en una escala } \\
\text { numérica del } 1 \text { al } 10 \text { al ingreso, a la } \\
\text { llegada a la Unidad disminución de la } \\
\text { intensidad del dolor a 4). }\end{array}$ & $\begin{array}{l}\text { [1605] Control del dolor. } \\
\text { Escala: desde } 1 \text { nunca demostrado a } 5 \\
\text { siempre demostrado. } \\
\text { - [160501] Reconoce los factores cau- } \\
\text { sales primarios (P. Inicial: 2; P. Final: } \\
4 \text { en una semana). } \\
\text { - [160503] Utiliza medidas preventivas } \\
\text { para el control el dolor (P. Inicial:2; P. } \\
\text { Final: } 4 \text { en una semana). }\end{array}$ & $\begin{array}{l}\text { [2150] Terapia de diálisis peritoneal. } \\
\text { - Valorar la permeabilidad del catéter, } \\
\text { observando la dificultad del flujo de } \\
\text { entrada/salida. Se realizan lavados } \\
\text { de } 500 c c \text { con una infusión de } 1500 c c \\
\text { de Physioneal } 1,36 \% \text { de Baxter }{ }^{\circledR} \text { sin } \\
\text { incidencias en infusión y drenaje. } \\
\text { - Observar si hay signos de infección } \\
\text { (peritonitis e inflamación/drenaje del } \\
\text { sitio de salida). No se observan signos } \\
\text { de peritonitis tras valoración del } \\
\text { drenaje (no dolor abdominal, efluente } \\
\text { claro sin turbidez, afebril). } \\
\text { - Observar si hay signos de dificultad } \\
\text { respiratoria. Sin alteraciones en la } \\
\text { frecuencia respiratoria, saturación de } \\
\text { oxígeno óptima monitorizada median- } \\
\text { te pulsioxímetro, se decide reducir el } \\
\text { volumen de infusión de cada ciclo a } \\
\text { 1800cc para disminuir la plenitud que } \\
\text { le genera el tratamiento. }\end{array}$ \\
\hline $\begin{array}{l}\text { [00126] Conocimientos deficientes. } \\
\text { Característica definitoria: } \\
\text { - No sigue completamente las instruc- } \\
\text { ciones. } \\
\text { Factor relacionado: } \\
\text { - Información insuficiente. }\end{array}$ & $\begin{array}{l}\text { [1814] Conocimiento: procedimiento } \\
\text { terapéutico. } \\
\text { Escala: de } 1 \text { ningún conocimiento a } 5 \\
\text { conocimiento extenso. } \\
\text { - [181404] Uso correcto del equipa- } \\
\text { miento. (P. Inicial: } 3 ; \text { P. Final: } 5 \text { en } \\
\text { una semana) } \\
\text { - [181410] Posibles efectos indesea- } \\
\text { bles: factores relacionados, signos } \\
\text { y síntomas y consecuencias de la } \\
\text { aparición de un neumoperitoneo. (P. } \\
\text { Inicial: } 2 \text {; P. Final: } 4 \text { en una semana). }\end{array}$ & $\begin{array}{l}\text { [2150] Terapia de diálisis peritoneal. } \\
\text { - Enseñar el procedimiento al paciente } \\
\text { que requiera diálisis domiciliaria: se } \\
\text { procede a un reentrenamiento con el } \\
\text { paciente tras la identificación de los } \\
\text { errores cometidos durante la técnica. } \\
\text { El paciente reconoce no cerrar las } \\
\text { pinzas del equipo de líneas antes de } \\
\text { la conexión de estas a las bolsas de } \\
\text { diálisis y no comprobar que la línea } \\
\text { del paciente está totalmente purgada } \\
\text { antes de iniciar la terapia. }\end{array}$ \\
\hline $\begin{array}{l}\text { [00073]Afrontamiento familiar incapa- } \\
\text { citante. } \\
\text { Características definitorias: } \\
\text { - Hiperatención prolongada al paciente } \\
\text { Factores relacionados: } \\
\text { - Estilos de afrontamiento diferentes } \\
\text { entre la persona de referencia (familia) } \\
\text { y el paciente. }\end{array}$ & $\begin{array}{l}\text { [2600] Afrontamiento de los problemas } \\
\text { de la familia. } \\
\text { Escala: de } 1 \text { nunca demostrado a } 5 \text { siem- } \\
\text { pre demostrado. } \\
\text { - [260007] Expresa sentimiento y } \\
\text { emociones abiertamente entre los } \\
\text { miembros. (P. Inicial: 3; P. Final: } 4 \text { en } \\
\text { un mes) } \\
\text { - [260021] Utiliza estrategia para con- } \\
\text { trolar el conflicto familiar (P. Inicial: } \\
\text { 2; P. Final: } 3 \text { en un mes) }\end{array}$ & $\begin{array}{l}\text { [ } 7140 \text { ] Apoyo a la familia. } \\
\text { - Valorar la reacción emocional de la } \\
\text { familia frente a la enfermedad del } \\
\text { paciente. } \\
\text { - Favorecer una relación abierta de } \\
\text { confianza con la familia. } \\
\text { - Crear un ambiente terapéutico de } \\
\text { apoyo para la familia. } \\
\text { - Aceptar los valores familiares sin } \\
\text { emitir juicios. } \\
\text { - Responder a todas las preguntas de } \\
\text { los miembros de la familia o ayudar- } \\
\text { les a obtener las respuestas. } \\
\text { - Escuchar las preocupaciones, senti- } \\
\text { mientos y preguntas de la familia. }\end{array}$ \\
\hline
\end{tabular}


en el año 1996, con características similares a nuestro paciente. En el artículo se describe como la paciente no conectó adecuadamente la bolsa de drenaje y al no realizar un drenaje inicial la maquina procedió a la infusión de aire en el peritoneo, provocando el neumoperitone $0^{8}$. Podemos afirmar que esta complicación es más frecuente encontrarla en pacientes en terapia con DPCA, esto es debido por los errores durante la realización de la técnica ${ }^{2-7}$.

Resulta fundamental proporcionar la educación y el entrenamiento necesario para una correcta realización de la terapia en DPA, dando importancia a la fase de purgado del sistema para evitar complicaciones. Es importante que los pacientes conozcan las causas de las complicaciones y reflexionen sobre cuales han podido ser los fallos potenciales para proceder a un reentrenamiento con una enfermera con formación en peritoneal.

La descripción de este tipo de casos servirá para evitar complicaciones en otros pacientes y que se tenga en cuenta durante los entrenamientos el tipo de terapia de cada paciente y las posibles complicaciones que puede conllevar la técnica. En definitiva, podemos concluir la importancia de individualizar la formación de los pacientes renales en función del tipo de terapia prescrita.

Recepción:15-06-20

Aceptación: 4-11-20

Publicación: 30-12-20

\section{Bibliografía}

1. Paniagua Sierra JR, Ventura García MJ, Vázquez Hernández R. Aumento de la presión intraabdominal y otras complicaciones. En Montenegro Martínez J, Correa Rotter R, Riella MC. Tratado de diálisis peritoneal. Barcelona: $2^{a}$ edición; 2015. p. 321-331.

2. Imran $M$, Bhat $R$, Anijeet $H$. Pneumoperitoneum in peritoneal diálisis patients; one centre's experiencie. NDT Plus. 2011;4(2):120-3.

3. Saunders RN, Veitch PS, Nicholson ML. Pneumoperitoneum in CAPD peritonitis. J R Soc Med. 2004;97(1):28-9.

4. Chen YC. Peritoneal dialysis-related peritonitis with Klebsiella pneumoperitoneum mimicking viscus perforation. Perit Dial Int. 2012;32(5):575-7.

5. Durán-Muñoz MI, Martín-Santiago C, de la Cierva-Medina $P$, Lope-Andrea $T$ y Martín-Acero $T$. Neumoperitoneo en pacientes con tratamiento renal sustitutivo con diálisis peritoneal continua ambulatoria. Enferm Nefrol. 2013;16(3):196-8.

6. Pérez Díaz V, Oviedo Gómez V, Fernández Carbajo B, Fernández Arroyo L, Martín Alcón B. Long-term pneumoperitoneum in continuous ambulatory peritoneal dialysis (CAPD) caused by handling fault of Stay.Safe ${ }^{\circledR}$ system associated to bicaVera solution. NDT Plus. 2011;4(3):195-7.

7. Chen WL, Tsao YT. Blunt abdominal trauma: a hidden culprit. NDT Plus. 2009;2(2):171-2.

8. Albalate $M$, Alcázar $R$, Ortiz A, Reyero A, M, Casado S, Caramelo C. Acute Pneumoperitoneum in a Patient on Nocturnal Intermittent Peritoneal Dialysis. Nephron. 1996;72:358.

9. Herramienta online para la consulta y diseño de Planes de Cuidados de Enfermería. [Internet]. NNNConsult. Elsevier; 2015 [consultado 3 marzo 2020]. Disponible en: https://www.nnnconsult.com.

Este artículo se distribuye bajo una Licencia Creative Commons Atribución-NoComercial 4.0 Internacional. https://creativecommons.org/licenses/by-nc/4.0/ 